Im Journal Club dieser Ausgabe der pädiatrie hautnah referieren und kommentieren diese Experten für Sie Arbeiten aus der internationalen Fachliteratur.
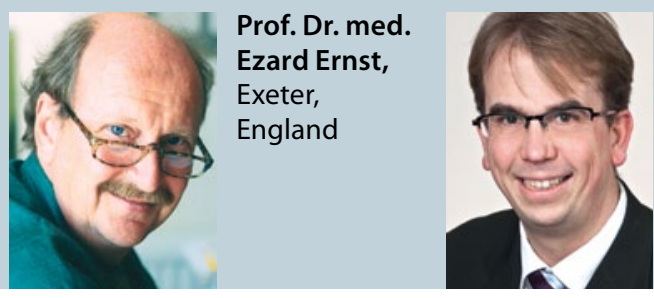

Prof. Dr. med. Helge Frieling, Hannover

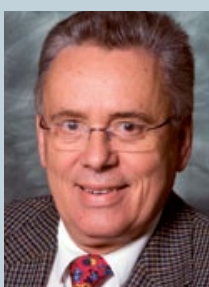

Dr. med. Hartmut Koch, Vechta

\title{
Nichtraucherschutz führt zu weniger Frühgeborenen
}

Es gibt zahlreiche Hinweise darauf, dass sowohl aktives als auch passives Rauchen das Risiko von Schwangerschaftskomplikationen erhöht. In Schottland, wo schon 2006 ein Rauchverbot eingeführt wurde, hat man nun die Auswirkungen auf die Raten an Frühgeborenen und SGA-Kindern genauer unter die Lupe genommen.

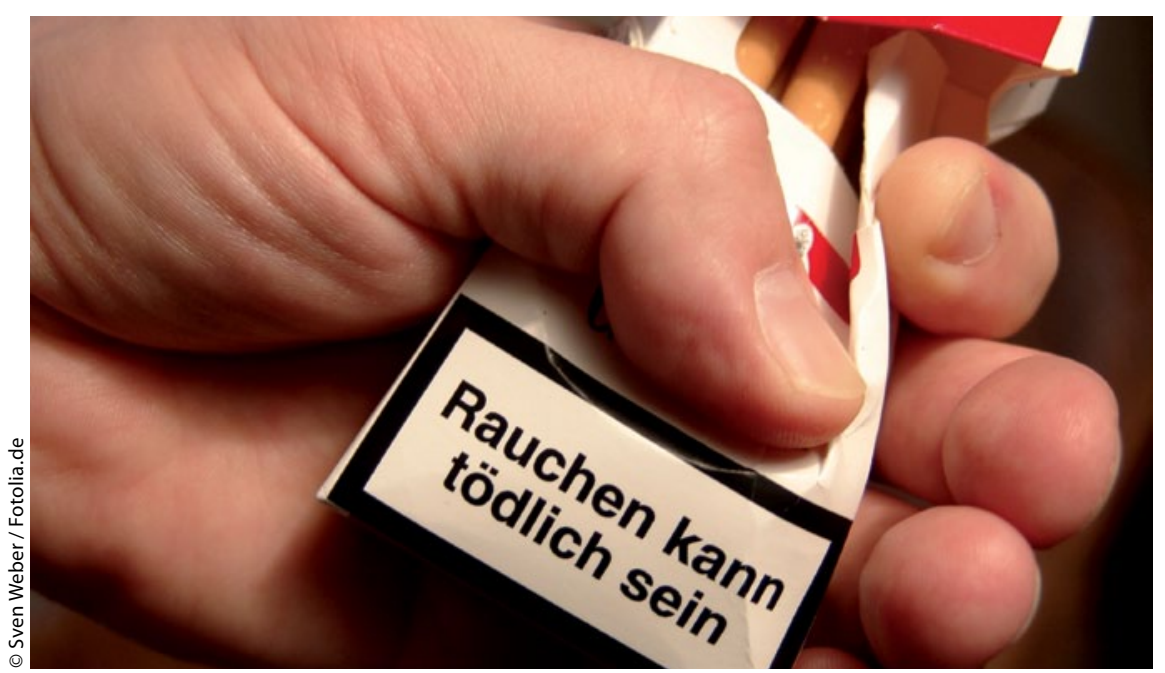

In Schottland kamen dank des Rauchverbots weniger Frühchen und SGA-Kinder zur Welt.

n Schottland wurde im März 2006 per Gesetz das Rauchen in allen geschlossenen, der Öffentlichkeit zugänglichen Räumen und Arbeitsplätzen verboten. Dieses Gesetz erwies sich als sehr gut durchsetzbar: Bereits nach zwei Wochen kam es zu einer Reduktion der Feinstaubkonzentration (PM2,5) um $86 \%$ in öffentlich zugänglichen Räumen. Die Befürchtung, dass als Folge der Gesetzgebung nunmehr vermehrt zuhause geraucht würde, hatte sich nicht bestätigt.
Dagegen konnte gezeigt werden, dass es zu einer signifikanten Verminderung von Herz-Kreislauf- und Atemwegserkrankungen auf Populationsniveau kam.

In einer weiteren Studie von der Universität Glasgow wurden nun die Auswirkungen dieses Gesetzes auf die Häufigkeit von Frühgeborenen und SGAKindern („small for gestational age“) untersucht. In einem Land mit einem staatlichen Gesundheitssystem liegt hierzu eine außergewöhnlich günstige Lage zur
Datenerfassung vor. So wurden die Daten von 716.941 Frauen, die in der Zeit zwischen August 1995 und Februar 2009 schwanger wurden und daraufhin einen lebenden Einling zwischen der 24. und 44. Schwangerschaftswoche geboren hatten, in die Untersuchung aufgenommen. In dem Zeitraum fiel die Prävalenz des Rauchens von $25,4 \%$ vor der Gesetzgebung auf $18,8 \%$ nach der Gesetzgebung

Das Rauchverbot selbst hatte einen antizipatorischen Effekt: Die Häufigkeit von Frühgeburten und Geburten von SGA-Kindern war bereits drei Monate vor Inkrafttreten des Gesetzes rückläufig. Der Rückgang bei den Frühgeborenen betrug $11,72 \%$, bei den SGA-Kindern $4,52 \%$. In der Subgruppenanalyse zeigte sich, dass die signifikanten $\mathrm{Re}$ duktionen sowohl bei Müttern, die noch rauchten, als auch bei solchen, die niemals geraucht hatten, zu beobachten war.

Mackay DF et al. Impact of Scotland's smokefree legislation on pregnancy complications: retrospective cohort study. PLoS Med 2012; 9: e1001175

Kommentar: Es gibt eine Gruppe von Rauchern, die in höchst aggressiver Weise ihr angebliches Recht auf Toleranz des Rauchens durch Nichtraucher zu verteidigen versucht. Diese Untersuchung macht wiederum klar, dass es einen Anspruch auf Toleranz gegenüber einer Körperverletzung nicht geben kann. Erst jüngst wurde erneut gezeigt, dass die Verhinderung elterlichen Rauchens eine der wichtigsten Maßnahmen zur Asthmaprävention bei Kindern ist [Burke $\mathrm{H}$ et al. Pediatrics 2012; 129: 735-44].

Dr. Hartmut Koch 